1 Institute of Mental Health, University College London, London

Cite this as: $B M J$ 2021;375:n3109 http://dx.doi.org/10.1136/bmj.n3109 Published: 17 December 2021

\title{
Long covid: research must guide future management
}

\section{Anthony S David professor of mental health}

Back in February 2021, the UK government announced that $£ 18.5 \mathrm{~m}$ is being invested into clinical research into long covid by UK Research and Innovation (UKRI). ${ }^{1}$ In light of public and medical concern about the long term effects of covid-19 infection, this is welcome.

In 1988, colleagues and I published an article in The $B M J$ called "Post viral fatigue: time for a new approach." "This was an attempt by young researchers to make the case that viewing the syndrome through a more unified mental-physical health lens and epidemiological orientation might counteract the increasingly polarised debate between patients with post-viral fatigue or myalgic encephalomyelitis (ME) and their doctors. We called for a clearer operational case definition, a biopsychosocial approach, and an end to emotive language all round. A generation later and our calls remain current.

Bodies including the National Institute for Health and Clinical Excellence (NICE), the Scottish Intercollegiate guidelines network (SIGN), the Royal College of General Practitioners (RCGP) and the Centres for Disease Control and Prevention (CDC) in the US have all attempted to operationally define persistent symptoms and disability after acute covid-19 illness. ${ }^{3}$ I think the term used by NICE-post-covid-19 syndrome-is a reasonable starting point. NICE's definition includes the phrase "not attributable to alternative diagnoses." This seems obvious, and yet is critical, as this should not be a catch-all category where any disorder with unexplained symptoms can be attached. For example, if a patient recovers from the acute respiratory illness, but remains short of breath and is found to have pulmonary fibrosis or pericarditis by accepted criteria, or, experiences brain fog and mental slowing, later linked to microvascular infarcts on MRI-can they be removed from the post-covid-19 cohort? I would say yes. Their condition may be unusual, and it may be serious, but it is not mysterious. These conditions add to the tally of morbidity caused by covid-19, but not to post-covid-19 syndrome. This, however, depends on patients getting access to the right assessments and investigations and there have been many calls from long covid patients to ensure that they have proper medical assessment of their symptoms.

We must not fall into the trap set by all "unexplained" symptoms: endless investigation by clinicians who then impart the "good news" that the test was normal. Because there is no accompanying positive explanation for the symptoms, this is perceived as implying, "it's not real" or somehow worse, that "it's all in your mind.” A devastating and embittering experience for many patients whose symptoms persist, and ultimately unhelpful in reaching a solution.

One problem facing us is how best to help those who, after a reasonable round of specialist assessments, do not have an alternative diagnosis or an identifiable explanation for their condition. Reaching a shared understanding on what a "reasonable" round of investigations consists of is a critical next step. New research is therefore welcome, and will hopefully provide algorithms and minimum standards for such specialist assessments, help us gain a better understanding of the mechanisms, treatments, and care pathways for long covid, and develop a robust evidence base.

It is no surprise that covid-19 has become the focus for a renewed debate about post-viral fatigue. It happens every time a pathogen is discovered or re-discovered. Indeed back in 1988 it was the coxsackie virus that was attracting attention, with chronic brucellosis and Epstein-Barr virus and Lyme disease not far behind. While a novel coronavirus, mankind's experience of which is less than two-years old, may be capable of all sorts of unique medical harms, we should not lose sight of the variety of previous pathogens which have plausibly led to long term sequelae (especially fatigue). This suggests that for them to all lead to this outcome the mechanisms may be general and indirect and not unique to SARS-CoV-2.

Emerging evidence suggests that long-covid is as likely to follow mild and even unconfirmed infection as severe illness requiring hospitalisation and respiratory support, although population-based studies on non-hospitalised cases are required to complete the picture. ${ }^{4} \mathrm{New}$ preliminary data suggest that people admitted to hospital with covid-19 who reported long covid symptoms had made only limited improvement after a full year. ${ }^{5}$ The demographic risk factors for covid-19 illness: age, male sex, ethnic minority status, underlying medication conditions, etc., do not seem to apply to post-covid syndrome. ${ }^{6}$ This all points to the need to improve our understanding of post-covid syndrome. UKRI-funded research using established clinical databases and longitudinal cohorts should seek to clarify these questions.

Will the new long-covid multidisciplinary teams be the setting of a never-ending journey round in circles or will they have the courage to accept uncertainty? Increasing evidence shows organ damage and pathological changes post covid, and we must be aware of many patients who have severe and debilitating fatigue and have not been believed or offered much help at all. But we must also remain open minded that some multi-symptom presentations may not be due to hitherto unidentified multi-system pathologies (with negative investigations), but a 
singular mechanism of heightened awareness of bodily sensations and objective malfunctions. ${ }^{7}$ It is a hypothesis and therefore provisional, but if articulated well in a clinical setting, with humility yet optimism, can lead to agreed new ways forward, which may be beneficial.

The injection of funding into this area will hopefully help guide future management of a large number of people with long covid-19 and, with luck, might even help many more with the broad range of post-viral conditions.

Competing interests: none declared.

Provenance and peer review: not commissioned, peer reviewed.

1 https://www.ukri.org/news/18-5-million-to-tackle-long-covid-in-the-community/

2 David AS, Wessely S, Pelosi AJ. Postviral fatigue syndrome: time for a new approach. Br Med J (Clin Res Ed) 1988;296:696-9. doi: 10.1136/bmj.296.6623.696 pmid: 3128374

3 Shah W, Hillman T, Playford ED, Hishmeh L. Managing the long term effects of covid-19: summary of NICE, SIGN, and RCGP rapid guideline. BMJ 2021;372:n136. doi: 10.1136/bmj.n136 pmid: 33483331

4 Altmann DM, Boyton RJ. Decoding the unknowns in long covid. BMJ2021;372:n132. doi: 10.1136/bmj.n132 pmid: 33541867

5 Evans RA, Leavy OC, Richardson M, et al. Clinical characteristics with inflammation profiling of long-covid and association with one-year recovery following hospitalisation in the UK: a prospective observational study. medRxiv 2021 Dec 15 [preprint]. https://www.medrxiv.org/content/10.1101/2021.12.13.21267471V1doi:10.1101/2021.12.13.21267471doi:10.1101/2021.12.13.21267471

6 Davis HE, Assaf GS, McCorkell L, etal. Characterizing long COVID in an international cohort: 7 months of symptoms and their impact. EClinicalMedicine 2021;38:101019. doi: 10.1016/j.eclinm.2021.101019. pmid: 34308300

7 Maxwell E. Unpacking post-covid symptoms. BMJ2021;373:n1173. doi: 10.1136/bmj.n1173. pmid: 34011496

8 Sudre CH, Murray B, Varsavsky T, et al. Attributes and predictors of Long-COVID: analysis of COVID cases and their symptoms collected by the Covid Symptoms Study App. medRxiv preprint doi: 10.1101/2020.10.19.20214494. 